\title{
A REVIEW ARTICLE-GESTATIONAL DIABETES MELLITUS
}

\author{
MUHAS C. ${ }^{*}$, NASEEF P. P. \\ Department of Pharmacy Practice, Jamia Salafiya Pharmacy College, Pulikkal, Malappram, 673639 \\ Email: muhasc@mail.com
}

Received: 21 Sep 2016, Revised and Accepted: 15 Nov 2016

\begin{abstract}
Diabetes mellitus is a chronic illness that requires continuing medical care and ongoing patient self-management education and support to prevent acute complications and to reduce the long-term complications. Moderate to severe maternal hyperglycemia in pregnancy has unique diabetesrelated risks to mother and her unborn baby. So Gestational Diabetes mellitus (GDM) is a carbohydrate intolerance that is not diabetes that has developed or been discovered for the first time during pregnancy. Approximately $7 \%$ of pregnancies are affected by GDM. Patient with GDM are at higher risk for excessive weight gain, preeclampsia, and cesarean sections. Infants born to mothers with GDM are at higher risk for macrosomia, birth trauma, and shoulder dystocia. After delivery, these infants have a higher risk of developing hypoglycemia, hypocalcemia, hyperbilirubinemia, respiratory distress syndrome, polycythemia and subsequent obesity and type 2 diabetes. So the management of GDM is very important, and its management remains a challenge for the obstetricians and endocrinologists. MNT is the most common therapy which suffices for GDM, but when required. The pharmacological treatment becomes necessary, and the treatment of choice is human insulin. OHAs have also reached the high tables in the management of GDM. Glyburide and metformin have been found to be safe, effective and economical for the treatment of gestational diabetes. Let us join hands to manage the GDM effectively, not only for the present generation but also for the generations to come.
\end{abstract}

Keywords: Gestational diabetes mellitus, GDM

(C) 2016 The Authors. Published by Innovare Academic Sciences Pvt Ltd. This is an open access article under the CC BY license (http://creativecommons.org/licenses/by/4.0/) DOI: http://dx.doi.org/10.22159/ijcpr.2017v9i1.16615

\section{INTRODUCTION}

Diabetes mellitus is a chronic illness that requires continuing medical care and ongoing patient self-management education and support to prevent acute complications and to reduce the long-term complications. Diabetes care is complex and requires multifactorial risk reduction strategies beyond glycemic control. A large body of evidence exists that support a range of interventions to improve diabetes outcomes [1].

Moderate to severe maternal hyperglycemia in pregnancy has unique diabetes-related risks in that there are potential long-term consequences for two individuals rather than just one: the mother and her unborn baby. Gestational diabetes(GDM) has been defined as any degree of glucose intolerance with onset or first recognition during pregnancy, to differentiate it from pre-diagnosed type 1 or type 2 diabetes or maturity-onset diabetes of the young (MODY) in women that get pregnant. This broad definition of GDM there for includes women whose glucose intolerance develop during pregnancy and who's that had preexisting diabetes which had not been diagnosed before pregnancy. The distinction here is important as, unlike most unborn babies of women who develop glucose intolerance in pregnancy, those of women with pre-existing diabetes can be exposed to hyperglycemia in the first two trimesters of pregnancy resulting in an increased risk of a range of both isolated and multiple cardiovascular and other abnormalities (including central nervous system and musculoskeletal defects). To try and clarify the situations, the consensus panel of the international association of diabetes and pregnancy study groups (IADPSG) recently recommended that highrisk women found to have diabetes at their initial prenatal visit should be diagnosed as having over diabetes rather than GDM [2].

The basic cause of type 2 diabetes, whose prevalence is rapidly increasing worldwide, is genetic factors with the addition of such acquired factors as lack of exercise, obesity caused by a high-fat diet, stress and aging impairing insulin action, leading to the onset of diabetes. In Japan, there is a clear trend towards delayed marriage and childbirth, and in future, the number of women with decreased carbohydrate tolerance who develop gestational diabetes mellitus during pregnancy is expected to increase more and more. It is a fact that it is known that the incidence of GDM increases by approximately 8 times for pregnant women age 35 y and over compared with women aged 25 y or under.

Originally GDM was defined as decreased carbohydrate tolerance that develops or is first identified during pregnancy, but in 2010 the definition was changed as following. Thus, GDM is a carbohydrate intolerance that is not diabetes that has developed or been discovered for the first time during pregnancy. The GDM definition there for does not include overt diabetes in pregnancy. Accordingly, hyperglycemic disorders that are excluded from the thought to have been overlooked until the pregnancy are excluded from the definition of GDM and are instead diagnosed as "overt diabetes in pregnancy" [3].

Approximately $7 \%$ of pregnancies are affected by GDM. It can be much higher incidence in certain minority populations, with a greater predisposition to diabetes. Patient with GDM are at higher risk for excessive weight gain, preeclampsia, and cesarean sections. Infants born to mothers with GDM are at higher risk for macrosomia, birth trauma, and shoulder dystocia. After delivery, these infants have a higher risk of developing hypoglycemia, hypocalcemia, hyperbilirubinemia, respiratory distress syndrome, polycythemia and subsequent obesity and type 2 diabetes [4].

\section{Historic evolution}

The history of GDM dated back to 1964 when O'Sullivan proposed specific criteria to interpret the glucose tolerance level in pregnancy to identify women at a higher risk for developing diabetes after delivery. The criteria were later modified by the National Diabetes Data Group (NDDG) in 1979. In 2008, the result of "Hyperglycemia and Adverse Pregnancy Outcomes (HAPO)" study was published. This major observational study provided us valuable information regarding the risks of adverse outcomes associated with various degrees of maternal glucose intolerance [5].

\section{Epidemiology}

The quoted prevalence of GDM ranged from 1 to 14\%. It depended on which population was being studied and which screening strategies and diagnostic criteria were used. The prevalence in the United Kingdom, US, and among European countries was estimated to be $5 \%, 3-7 \%, 2-6 \%$ respectively. Higher prevalence of GDM was 
noted in African, Asian, Indian and Hispanic women. Once a disease of older people, type 2 diabetes was increasingly affecting women during their fertile years, many population studies indicated that the increasing incidence of GDM parallels that of its type 2 group. Together with the new diagnostic criteria which included more patients with a lesser extent of hyperglycemia and increasing rate of obesity, the prevalence would continue to rise [5].

\section{Risk factors}

(1) The age increased risk with increasing age; $<25$ y old considered low risk.

(2) Ethnicity:

- Highest risk-Native Americans, Hispanic, Asian decent

- Moderate risk-African women

- Lowest risk-Non-Hispanic white women

(3) Obesity

(4) Family history

(5) Past obstetric history

- Women who have previously delivered a large infant

- Women with a history of previous GDM [6]

\section{Pathophysiology}

Pregnancy is a condition characterised by progressive insulin resistance that begins near mild pregnancy and progressive through the third trimester. In late pregnancy, insulin sensitivity is fallen by $\sim 50 \%$. Two main contributors to insulin resistance include increased maternal adiposity and insulin desensitising effects of hormones produced by the placenta. The fact that insulin resistance rapidly decreases post-delivery suggests that the major contributors are placental hormones.

The placenta produces human chorionic somato-mammotrophs (HCS, formerly called human placental lactogen), bound and frees cortisol, estrogen, and progesterone. HCS stimulates pancreatic secretion of insulin in the fetus and inhibits peripheral uptake of glucose in the mother. As the pregnancy progresses and the size of the placenta increases, so does the production of the hormones, leading to a more insulin-resistant state. In nondiabetic pregnant women, the first and second phase insulin responses compensate for this reduction in insulin sensitivity, and this is associated with betacell hypertrophy and hyperplasia. However, women have a deficit in this additional insulin secretory capacity develop GDM. Beta-cell dysfunction in women diagnosed with GDM may fall into one of three major categories:

(1) Autoimmune, (2) monogenic, or occurring on a background of insulin resistance (as is most common) [4].

\section{Diagnosis}

When diagnosing GDM, clinicians must keep in mind that patients may in fact have (1) undiagnosed type 2 diabetes, (2) mild abnormal glucose tolerance before pregnancy that worsens in pregnancy because of increased insulin resistance, (3) normal glucose tolerance before pregnancy that become abnormal with advancing gestation, or (4) undiagnosed type 1 diabetes when pregnancy coincides with the prodromal phase of type 1 diabetes (rare) [4].

One study described five screening criteria to determine whether a patient is presenting with latent autoimmune diabetes in adults (LADA). These include; (1) age of diabetic onset $<50 \mathrm{y}$, (2) acute symptoms of polyuria, polydipsia, and or weight loss, (3) personal history of autoimmune disease, (4) family history of autoimmune disease; and (5) $\mathrm{BMI}<25 \mathrm{~kg} / \mathrm{m}^{2}$.

The following diagnostic criteria are based on the 1998 clinical practice guidelines for the management of diabetes in Canada [7].

\section{Fasting or random blood sugars}

A fasting glucose level $>126 \mathrm{mg} / \mathrm{dl}$ or a random plasma glucose $>200$ $\mathrm{mg} / \mathrm{dl}$ meets the threshold for the diagnosis of gestational diabetes mellitus.

\section{Testing with 75g 0GTT}

The 75g $2 \mathrm{~h}$ OGTT is the diagnostic test recommended by the WHO. Table 1 summarises the diagnostic criteria for the $75 \mathrm{~g}$ OGTT are proposed by several medical organisations.

The 100 g OGTT: in North America, a 100 g glucose load may be used in diagnosing GDM.

Table 1: Criteria for diagnosis of GDM with the 75g OGTT

\begin{tabular}{|c|c|c|c|c|}
\hline Organization & Fasting & $1 \mathrm{~h} \mathrm{PG}$ & 2 h PG & Diagnostic criteria for GDM \\
\hline WHO & $>126 \mathrm{mg} / \mathrm{dl}$ & Not measure & $\geq 140 \mathrm{mg} / \mathrm{dl}$ & One abnormal value \\
\hline $\begin{array}{l}\text { Clinical practice guidelines for the management of } \\
\text { diabetes in canada }\end{array}$ & $\geq 95 \mathrm{mg} / \mathrm{dl}$ & $\geq 190 \mathrm{mg} / \mathrm{dl}$ & $>160 \mathrm{mg} / \mathrm{dl}$ & $\begin{array}{l}\text { Two or more abnormal } \\
\text { value }\end{array}$ \\
\hline
\end{tabular}

\section{Complications}

1) Increased risks of maternal complications: in the case of women with diabetic complications, during pregnancy, there are risks that diabetic retinopathy and diabetic nephropathy could deteriorate and ketoacidosis could increase.

2) Increased risks of obstetric complications: Increased incidence of pregnancy-induced hypertension, caesarean section, bradytocia due to macrosomia, and induction of childbirth.

3) Increased risks of perinatal complications: increased incidence of macrosomia, delayed intrauterine fetal development, neonatal complications (hypoglycemia, polycythemia, hyperbilirubinemia, etc.)

4) In the case of GDM, there is an increased risk of the mother developing type 2 diabetes in future.

5) The incidence of developing lifestyle-related diseases in offsprings of mothers with GDM [3].

\section{Management}

The aim of treatment is to maintain maternal blood concentration within an acceptable range in a normal pregnancy [8]. Among women who have
GDM and normal fasting blood glucose levels, treatment, as necessary, reduces rates of adverse pregnancy outcomes including perinatal mortality, neonatal hypoglycemia, neonatal hyperbilirubinemia, elevated cord blood C-peptide level, and birth trauma [9].

Once a woman has been diagnosed with GDM, a decision must be made as to whether she should be treated and if so for how long. Women will be diagnosed late in her pregnancy and little time is usually remaining from the time of the GDM diagnosis to the time of delivery [10]. It is important to recognise that delay in therapy may result in irreversible adverse outcomes. Treatment for GDM can encompass three different therapies: dietary changes, exercise and pharmacotherapy [11].

Monitoring blood glucose at home is important to tailoring specific treatment and making adjustment as needed. ${ }^{4}$ Then it is followed by appropriate diet therapy which is extremely important during pregnancy, a pregnant women patient need to consume adequate energy, proteins and minerals [3].

\section{Medical nutrition therapy (MNT)}

All women with GDM should receive nutritional counselling. The meal pattern should provide adequate calories and nutrients to meet 
the needs of pregnancy [12]. MNT is the cornerstone of therapy for women with GDM. Specifically, the management of GDM entails calorie and nutrient restrictions and manipulations as a strategy to normalise blood glucose levels. The food plan should be designed preferably by a dietician so as to fulfil minimum nutrient requirements for pregnancy and to achieve glycemic goals without inducing weight loss or excessive weight gain. MNT is a selfmanagement therapy. Education, support, and follow-up are required to assist the women in making lifestyle changes essential to successful nutrition therapy [9].

To satisfy the various needs, the following dietary principles have been suggested as suitable for someone with GDM[13-15]: (i) eating regular small meals of slowly absorbed carbohydrate to maintain blood glucose concentrations; (ii) taking similar portions of carbohydrate at meals daily (especially if the GDM is not treated by insulin); (iii) allowing the consumption of a moderate amount of sugar-containing foods as long as it neither promotes hyperglycemia nor excessive weight gain; (iv) eating at least five portions of fruits and vegetables per day; (v) eating low-fat dairy foods and attempting to drink a pint of milk or its equivalent every day and meat/fish/poultry or alternatives; (vi) attempting to eat two portions of oily fish per week. In addition the recently revised guidelines of the UK National Institute for Health and Clinical Excellence for pregnant women with GDM suggest that that prepregnancy BMI is greater than $27 \mathrm{~kg} / \mathrm{m}^{2}$ should restrict their energy intakes to $105 \mathrm{~kJ} / \mathrm{kg}$ per day or less and combine this with moderate exercise of at least 30 min duration per day [16].

\section{Exercise}

For vast majority of pregnant women with gestational diabetes, exercise will be beneficial and recommended [17]. Exercise decreases peripheral insulin resistance and is an appropriate adjunctive therapy to diet for the GDM patient [18]. Patients are encouraged to participate in exercise 30 min several times weekly [19]. In women with relative contraindications, appropriate assessment and counselling it may be possible to allow exercise in pregnancy. With appropriate caution, women with chronic respiratory conditions, obesity and previous sedentary lifestyle can be introduced to an appropriate exercise programme during pregnancy that may motivate them to continue exercise after pregnancy.

Pregnant women frequently require modifications of their exercise regimen because of musculoskeletal or mechanical symptoms such as pubic symphysis dysfunction or back pain. The prescription of an upper body exercise program, for example, cycle ergometry or water based exercise programs can overcome these limitations. By including specific exercise advice in the management plan for gestational diabetes, it may help validate the women's request for the support in both the workplace and home to allow her to meet these recommendations. As gestational diabetes is a relatively common complication, it may be practical to offer supervised group exercise such as walking groups, antenatal exercise classes or aqua aerobics [17].

\section{Pharmacological treatment}

Pharmacologic therapy is most commonly instituted once diet and exercise have failed as evidenced by an abnormality in more than half of self-monitored glucose values or an abnormal value in those women tested weekly [20]. Several studies also concluded that women who do not meet established goals with diet and exercise alone have more favourable outcomes with pharmacological interventions [4]. For some women, especially those with a relatively severe form of the condition that develops earlier than in the third trimester, GDM cannot be adequately controlled by diet and exercise alone. In these cases, pharmacological treatment is required to reduce circulating maternal glucose concentrations and there for its associated risk of enhanced fetal growth and to have no adverse (for example, teratogenic) effects on the fetus if the drug used for treatment crosses the placenta [2]. So, the mainly used pharmacological treatment in the management of GDM are as follows.

\section{Insulin therapy}

In the case where the patients are unable to achieve glycemic control with diet and exercise, pharmacotherapy with insulin is recommended. One recommended threshold for initiation of pharmacologic therapy requires a fasting glucose $>95 \mathrm{mg} / \mathrm{dL}$ in addition to postprandial levels $>120 \mathrm{mg} / \mathrm{dl}$ for $2 \mathrm{~h}$ or $>140 \mathrm{mg} / \mathrm{dl}$ for 1 hour. Insulin therapy is safe and effective and is the gold standard against which other therapies for GDM are compared. Regular and NPH insulin, and the short-acting insulin analogues lispro (Humalog) and Aspart (Novolog) are considered safe for safe [10]

When the blood glucose levels are rising out of the target range, our own insulin production can be 'topped up' with an injection of insulin at the meal time. We may need to take insulin at one or all of our meals. Sometimes the insulin we produced in between our meals and overnight may also need to 'topped up'. This may require extra slower-acting insulin at bedtime. We will be advised when how much insulin we will need to take by the diabetes team [6].

Insulin treatment for women with GDM can be done on an outpatient basis. The insulin dosage is maternal weight based. One insulin regimen doses insulin at $0.7 \mathrm{units} / \mathrm{kg}$ actual body weight. This dosage is lower than for, non-GDM diabetic patients. This more conservative therapy is intended to prevent hypoglycemia. After the total daily dose is calculated, two-thirds of the doses is administered before breakfast (two-thirds NPH insulin and one-third regular insulin) and the remaining one-third is broken up into 2 different doses (one-half regular insulin before dinner and one-half NPH insulin at bedtime) [10].

In order to achieve the near-normal glycemia, a subcutaneous applicator of exogenous insulin should have similar pharmacologic properties as insulin secreted from the pancreas in healthy individuals. The more attempts have been made to remodel insulin molecule with the purpose of changing its pharmacokinetic properties into more favourable ones. By changing the primary amino acid sequence, changes in the tertiary structure can be achieved, which could influence the association of molecules as well as the duration of insulin-receptor binding, which can also lead to an increased mitogenic action of analogue. The insulin lispro can better manage postprandial hyperglycemia than regular human insulin without increasing the risk of hypoglycemia. In clinical studies, insulin as part given immediately before the meal was more successful than regular insulin $30 \mathrm{~min}$ prior to a meal in reducing postprandial hyperglycemia and number of nocturnal hypoglycemic episodes.

There is only one report on the use of glargine in pregnancy. The patient with type 1 had frequent, severe nocturnal hypoglycemic episodes and her NPH was substituted for glargine. The patient had previously been free from retinopathy, and it does not occur during pregnancy. One prospective nonrandomized study of 445 patients has shown a reduction in operative deliveries and birth trauma in women with gestational diabetes who are treated with insulin. However, the finding of this study remains to be demonstrated in an adequately powered RCT.

There are no specific studies declaring one type of insulin or a certain regimen as superior in affecting any perinatal outcomes. A common initial dosage is 0.7 units per kg per day, with one dose consisting of the total amount given in the morning and one dose consisting of one-third of the total amount given in the evening. One-third of each dose is given as regular insulin and the remaining two-third as NPH insulin. a recent study of 42 women with gestational diabetes supports the safety of very short-acting insulin lispro, which can be used with once-daily extended insulin ultra-lente. The simplest regimen that will control blood glucose level is the best [21].

\section{Oral hypoglycemic agents}

\section{Metformin}

Metformin is a tablet that has been used successfully for almost $40 \mathrm{y}$ to treat diabetes outside of pregnancy. It is increasingly being used during pregnancy as an alternative to or in addition to insulin [6].

Metformin improves insulin sensitivity and would be expected therefore to improve tolerance in pregnancy by reducing the physiological rise in insulin resistance that occurs during pregnancy [22]. The biguanide metformin during pregnancy has mostly been studied in the first $12 \mathrm{w}$ of gestation for patients with polycystic ovary syndrome (PCOS). Preliminary studies have shown that in 
women with PCOS, metformin may be safe and may reduce risk of miscarriage and development of GDM when used for the entire pregnancy. Metformin may also have a role in therapy for GDM; a multicenter trial is underway in Newzealand to address this questions [4].

Metformin works by allowing to work insulin more effectively, so a smaller amount of insulin will work better. This can mean that your own limited insulin production may be enough to regulate blood glucose levels with metformin to boost its action. Metformin can also been useful in addition to insulin injections. Using them together can keep insulin doses lower. This can help prevent excessive pregnancy weight gain and therefore improves pregnancy outcomes. Unlike insulin, metformin does pass across the placenta. There have been a number of studies to look at the safety of metformin in pregnancy and your diabetes doctor in the clinic will be happy to discuss this with you. Metformin is not an option for everyone.

There are also certain medical conditions or pregnancy complications that may mean insulin would be a better choice. Some people ( 2 out of every 10) experience side effects from taking metformin, such as stomach upsets. These can be minimised by starting on a low dose, and by building the dose up slowly and by taking the tablet with or immediately after food [6].

\section{Glyburide}

This sulfonylurea has been identified in the past several years as an alternative to insulin therapy for the treatment of GDM. Its primary action is to enhance insulin secretion. Glyburide does not significantly cross the placenta. Several studies have found that glyburide serves as a suitable alternative to insulin for treatment of GDM with similar perinatal outcomes. A survey performed by the American colleges of Obstetricians and gynaecologists found that $13 \%$ of Obstetricians and maternal-fetal medicine specialists were using glyburide as a first-line agent in the treatment of women with GDM who failed to achieve glucose control with diet. A disadvantage to taking glyburide is that it sometimes takes $>1 \mathrm{w}$ to observe the effect of titration. However, it is inexpensive and less invasive than insulin and has been found to be as effective as insulin therapy for GDM treatment [4].

Glyburide is a category C medication in pregnancy. Hypoglycemia may occur with all sulfonylureas, and glyburide is no exception. The incidence of hypoglycemia with glyburide ranges from 1 to $5 \%$. The most common adverse effects are gastrointestinal and (nausea, vomiting, dyspepsia) and dermatologic (pruritis, urticaria, erythema, and maculopapular eruptions). Elevations of liver function testes have been reported, but jaundice is rare. The overall incidence of adverse effects ranges from 3.2 to $4.1 \%$.

Identifying those women who might fail glyburide therapy in pregnancy is important when deciding medical therapy for the treatment of gestational diabetes. Conway in an observational trial to examine factors predicting failure of glyburide treatment in gestational diabetes found that among women with high FPG levels greater than or equal to $110 \mathrm{mg} / \mathrm{dl}, 24 \%$ failed to respond to glyburide. Studies focusing on the transfer of glyburide into the milk of lactating mother have been performed. There were no cases of neonatal hypoglycemia, and it appears that breastfeeding is safe in women receiving glyburide. However, the drug has not been endorsed by any decision-making body for routine use in GDM [9].

\section{Acarbose}

Acarbose has not been systemically analysed in the treatment of pregnant women with diabetes. There is a report from Mexico on six women in whom glycemic regulation was achieved with acarbose, and the pregnancies were complicated by deliveries of healthy babies. The potential unfavourable (although not proven) influence of acarbose on pregnancy could be due to the increased amount of starch in the bowels of the women treated with acarbose. The bacterial breakdown of starch leads to the accumulation of butyrate, which could increase the prostaglandin E secretion, with negative consequences on pregnancy.

Acarbose is poorly absorbed from the gastrointestinal tract, and two preliminary studies have suggested efficacy in reducing postprandial glucose excursions in GDM but with the expected high frequency of cramping. A small proportion of this drug may be absorbed systemically, and safety and potential transplacental passage have not been fully evaluated [9].

\section{Monitoring glycemic control}

The success of the treatment during GDM depends on the glycemic control maintained with a meal plan or pharmacological intervention. To know the effectiveness of treatment, monitoring of glycemic control is essential.

- Women with type 1 or type 2 diabetes need pre-pregnancy counselling to maintain blood glucose levels. For women with type 1 and type 2 diabetes who are already on treatment, intensive monitoring is required from the day one of conception. They will have to do self-monitoring of blood glucose or get the levels checked at a laboratory at least once a week to adjust the dosage of insulin.

- Once target blood glucose is achieved, women with GDM till the $28^{\text {th }}$ week of gestation require monitoring of both fasting and 2-h post-breakfast once a month and at other time of the day as the clinician decides.

- After the $28^{\text {th }}$ week of gestation, the monitoring should be more frequent, at least once in two weeks, if need be more frequently.

- After $32 \mathrm{w}$ of gestation, monitoring should be done once a week till delivery.

- In high-risk pregnancies, the frequency of monitoring may be intensified with SMBG.

- Continuous glucose monitoring devices are available, but this equipment needs special training, and furthermore is expensive. These devices may be useful in high-risk pregnancies to know the glycemic fluctuations and to plan proper insulin dosage [12].

\section{Pharmacist's role}

A diagnosis of GDM implies that the patient and baby will have an increased chance of complication before, during and after delivery and that future pregnancies are also more likely to be complicated by diabetes or GDM [23]. It is important for women with GDM to be proactively treated and followed after delivery to prevent or minimise unfavourable outcomes. Pharmacists can help educate the patient regarding the various pharmacotherapy options available to her. A diagnosis of GDM is understandably an additional complication when so much is already happening to the patient's body. The women will need guidance in blood glucose testing. It is important that we as pharmacists encourage the women with GDM to be compliant with recommendations for her diet, exercise, blood glucose monitoring and medications she needs to be taking. Well controlled glycemia is important for preventing serious medical problems for the mother and the fetus [10].

\section{CONCLUSION}

In the management of gestational diabetes, the clinical condition of individual cases needs to be identified over time, and in addition to the cooperation of family members, multidisciplinary management and treatment are provided by obstetricians, gynaecologists, nutritionists, paediatricians, cardiovascular specialists, nephrologists, ophthalmologists, and nursing staff.

Optimal management of GDM remains a challenge for the obstetricians and endocrinologists. MNT is the most common therapy which suffices for GDM, but when required. The pharmacological treatment becomes necessary, and the treatment of choice is human insulin. Rapid-acting insulin analogs are a possible alternative to regular human insulin. Data on long-acting analogs are very sparse, so these analogs cannot be recommended in pregnancy for the time being. From insulin to OHAs has been a great journey in GDM. OHAs have reached the high tables in the management of GDM. Glyburide and metformin have been found to be safe, effective and economical for the treatment of gestational diabetes. They are also found to be safe for infants who are breastfed. More studies are needed to identify the risks and benefits of these, and other, oral 
hypoglycemic drugs before they can be recommended as first-line drugs for the treatment of pre-gestational and gestational diabetes. However, they can definitely be used in situations where insulin administration is not feasible or not accepted by the patient

Optimal treatment of GDM requires a team effort on the part of obstetricians and endocrinologists; above all, what is required is an active and alert clinician. Let us join hands to manage the GDM effectively, not only for the present generation but also for the generations to come.

\section{CONFLICT OF INTERESTS}

\section{Declared none}

\section{REFERENCES}

1. Sanders of medical care in diabetes, American diabetes association, care diabetes journal org; 2013.

2. Clive J, Petry. Gestational diabetes: risk factors and recent advances in its genetics and treatment. $\mathrm{Br} \mathrm{J}$ Nutr 2010;104:775-87.

3. Takashi Sugiyama. Management of gestational diabetes mellitus. JMAJ 2011;54:293-300.

4. Jennifer M, Perkins MD, Julia P, Dunn MD, Shubhada M, Jagasia MD. Perspectives in gestational diabetes mellitus: a review of screening, diagnosis, and treatment. Clin Diabetes Res 2001;25:57-62.

5. Cheung KW, Wrong SF. Gestational diabetes mellitus update and review of the literature. Reproductive Sys Sexual Disord 2011;S:2.

6. Gestational Diabetes-Medication Treatment Options. Patient Information, Cambridge University Hospitals. NHS; 2016. p. 1-3.

7. Howard Berger MD, Joan Crane MD, Dan Farine MD. Screening for gestational diabetes mellitus. SOGC clinical practice guidelines; 2002. p. 121.

8. Dornhorst A, Frost G. The principles of dietary management of gestational diabetes: reflection on current evidence. J Hum Nutr Diet 2002;15:145-56.

9. Navneet Magon, V Seshiah. Gestational diabetes mellitus: noninsulin management. Indian J Endocrinol Metab 2011;15:284-93.

10. Karen von Koeckritz. Current management of Gestational Diabetes Mellitus. Von Koeckritz-Gestational Diabetes; 2014. p. 1-7.
11. Lange 0 . Oral antihyperglycemic agents for the management of gestational diabetes mellitus. Obstet Gynecol Clin North Am 2007;34:255-74

12. Balaji V, Seshiah V. Management of diabetes in pregnancy. JAPI 2011;59:108-12.

13. Abell DA. The significance of abnormal glucose tolerance in pregnancy. Br J Obstet Gynaecol 1979;86:214-21.

14. Hill A. Encouraging healthier lifestyles-4. Dietary advise for diabetes in pregnancy. J Fam Health Care 2007;17:117-9.

15. Dornhorst A, Frost G. The principles of dietary management of gestational diabetes: reflection on current evidence. J Hum Nutr Diet 2002;15:145-56.

16. National Institute for Health and Clinical Excellence, diabetes in pregnancy: management of diabetes and its complications from preconception to the postnatal period. Available from: http://www.nice.org.uk/nicemedia/pdf/DiabetesFullGuideline RevisedJULY2008.pdf. [Last accessed on 20 Aug 2016]

17. Herbert Groeller, Sandra Lowe, Anthony Worsley, Arthur Jenkins. Does exercise have a role in the management of gestational diabetes mellitus? Obstetric Med 2010;3:133-8.

18. Menato GSB, Signorile A. Current management of gestational diabetes mellitus. Expert Rev Obstet Gynecol 2008;3:73-91.

19. Kim C. Gestational diabetes: risks, management, and treatment options. Int J Womens Health 2010;2:339-51.

20. Gabriella P, Tara D Benjamin. Update on gestational diabetes. Obstet Gynecol Clin N Am 2010;37:255-67.

21. David KT, Stephen DR, Elizabeth GB. Management of gestational diabetes mellitus. Am Fam Physician 2003;68:1767-72, 1775-6.

22. Steve LH, Jyoti B, Antoinette J, Hassan S. Metformin treatment for gestational diabetes. Br J Diabetes Vasc Dis 2009;9:220-5.

23. Simmons D, McElduff A, McIntyre HD, Elrishi M. Gestational diabetes mellitus: NICE for the U. S.? A comparison of the American diabetes association and the American college of obstetricians and gynaecologists guidelines with the U. K. National Institute for Health and Clinical Excellence guidelines. Diabetes Care 2010;33:34-7.

\section{How to cite this article}

- Muhas C, Naseef PP. A review article-gestational diabetes mellitus. Int J Curr Pharm Res 2017;9(1):1-5. 